\title{
Post publication peer review
}

Public Domain

\section{Source}

Open Research Glossary

Standard peer review, but after a research article has been formally published. 